\title{
Feedback control of thermal lensing in a high optical power cavity
}

\author{
Y. Fan, ${ }^{1, a)}$ C. Zhao, ${ }^{1}$ J. Degallaix, ${ }^{1, b)}$ L. Ju, ${ }^{1}$ D. G. Blair, ${ }^{1}$ B. J. J. Slagmolen, ${ }^{1, c)}$ \\ D. J. Hosken, ${ }^{2}$ A. F. Brooks, ${ }^{2, d)}$ P. J. Veitch, ${ }^{2}$ and J. Munch ${ }^{2}$ \\ ${ }^{1}$ School of Physics, University of Western Australia, 35 Stirling Highway, Crawley, Western Australia 6009, \\ Australia \\ ${ }^{2}$ Department of Physics, The University of Adelaide, Adelaide, South Australia 5005, Australia
}

(Received 1 May 2008; accepted 25 August 2008; published online 1 October 2008)

\begin{abstract}
This paper reports automatic compensation of strong thermal lensing in a suspended $80 \mathrm{~m}$ optical cavity with sapphire test mass mirrors. Variation of the transmitted beam spot size is used to obtain an error signal to control the heating power applied to the cylindrical surface of an intracavity compensation plate. The negative thermal lens created in the compensation plate compensates the positive thermal lens in the sapphire test mass, which was caused by the absorption of the high intracavity optical power. The results show that feedback control is feasible to compensate the strong thermal lensing expected to occur in advanced laser interferometric gravitational wave detectors. Compensation allows the cavity resonance to be maintained at the fundamental mode, but the long thermal time constant for thermal lensing control in fused silica could cause difficulties with the control of parametric instabilities. (C) 2008 American Institute of Physics.
\end{abstract}

[DOI: $10.1063 / 1.2982239]$

\section{INTRODUCTION}

The first generation laser interferometric gravitational wave detectors, such as LIGO, ${ }^{1}$ VIRGO, ${ }^{2}$ GEO600, ${ }^{3}$ and TAMA, ${ }^{4}$ have approached their design sensitivity. The planned second generation detectors, such as Advanced LIGO, ${ }^{5}$ LCGT, $^{6}$ and AIGO, ${ }^{7}$ will improve the strain sensitivity to $h \sim 10^{-24} / \sqrt{\mathrm{Hz}}$. Such detectors are very likely to detect gravitational waves from known sources. To reach the required strain sensitivity, improvements include substantially increased optical power to reduce shot noise. For example in Advanced LIGO, as shown in Fig. 1, the input optical power will be increased to $125 \mathrm{~W}$ leading to $2.1 \mathrm{~kW}$ of power in the power recycling cavity and about $830 \mathrm{~kW}$ of power in the 4 km length Fabry-Pérot (FP) interferometer arm cavities. However, at such a high optical power, even very low levels of absorption in the core optics substrate and dielectric coatings induce significant thermal lensing. ${ }^{8}$ This thermal lensing causes wavefront distortion that would prevent the detector from achieving high sensitivity and could even lead to serious degradation of the operation of the interferometer. ${ }^{9-11}$

Various thermal compensation methods have been proposed and tested. Lawrence et al. ${ }^{12}$ demonstrated that a ring radiative heater, inserted near the test mass, could compensate gross axisymmetric thermal lensing effects by creating a negative thermal lens. A scanning $\mathrm{CO}_{2}$ laser beam was used to compensate the nonaxisymmetric wave-front distortion caused by the inhomogeneous absorption. ${ }^{13}$ Based on this research, a thermal compensation system including a servo-

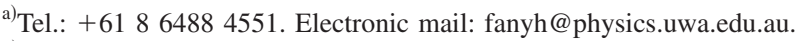

${ }^{b)}$ Present address: Albert Einstein Institute, GEO, Hannover, Germany.

${ }^{c}$ Present address: Centre for Gravitational Physics, the Australian National University, Canberra, Australia.

${ }^{d)}$ Present address: Caltech, Pasadena, California, USA.
}

system was developed for LIGO. It consists of a $10 \mathrm{~W} \mathrm{CO}_{2}$ laser that uses a mask with either a central spot or an annular pattern to obtain different heating modes. ${ }^{14,15}$

For proof-of-principle experiments in cylindrical symmetry, we chose to use a compensation plate (CP) encircled by a heating ring in close thermal contact with the circumference of the plate. The $\mathrm{CP}$ was made from fused silica. A nichrome heating wire was wrapped around its cylindrical surface. ${ }^{16,17}$ The experiment was performed at the Gingin high optical power facility ${ }^{18}$ (HOPF) of the Australian Consortium for Interferometric Gravitational Astronomy (ACIGA). The layout of the experimental setup is illustrated in Fig. 2. The CP is placed inside a FP cavity close to the input mirror $(\sim 150 \mathrm{~mm})$. Compensation occurs by creating an opposite thermal gradient matched to the laser beam induced thermal gradient in a test mass mirror. The FP cavity consists of two suspended sapphire mirrors, the input test mass (ITM) and the end test mass (ETM), which are separated by $\sim 80 \mathrm{~m}$. To enhance the thermal lensing effect, the ITM is reversed to have its high reflectivity coating on the outside. A high quality antireflective coating is placed on the inner surface of the ITM. The CP and the two mirrors are in vacuum, $\sim 10^{-6}$ mbar. A $\sim 4 \mathrm{~W}$ Nd:YAG (yttrium aluminum garnet) laser beam is injected and phase locked into the FP cavity using the Pound-Drever-Hall stabilization technique. ${ }^{19}$ The charge coupled device (CCD) camera, located behind the ETM, measures the transmitted beam profile leaking from the ETM. A lens images the plane of the ETM onto the CCD with a suitable demagnification to match the camera. A controllable de power supply applies heat to the CP. The controller is the key component in the closedloop control system. It is used to control the heating power according to an error signal derived from the transmitted beam spot size measured by the CCD camera. 


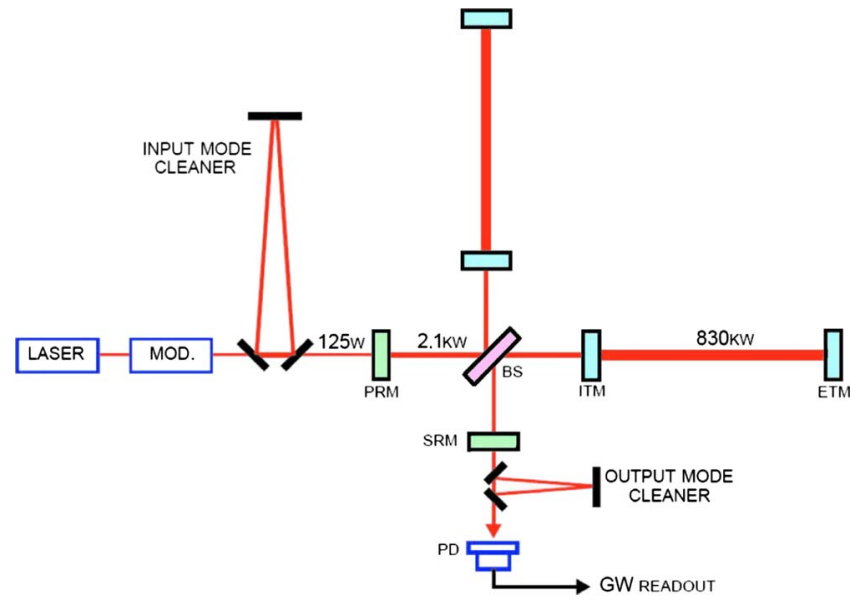

FIG. 1. (Color online) Layout of the Advanced LIGO interferometric detector. BS represents the beam splitter, and ITM represents input test mass; ETM, end test mass; PRM, power recycling mirror; and SRM, signal recycling mirror. The incident laser power is $125 \mathrm{~W}$, leading to about $2.1 \mathrm{~kW}$ in the power recycling cavity and $830 \mathrm{~kW}$ in the arm cavities.

In this paper we present the results of applying a feedback control system to automatically compensate the effect of thermal lensing. In Sec. II, we discuss the simulation of heating the $\mathrm{CP}$ using finite element modeling. We show the time evolution of the thermal focal length of the CP after applying a constant heating power. In Sec. III we discuss the experimental results and their comparison with the results from the simulation of the control loop.

\section{SIMULATION OF HEATING THE COMPENSATION PLATE}

The substrate of the CP is made from fused silica which has very low optical absorption $(\sim 2 \mathrm{ppm} / \mathrm{cm})$ and high homogeneity. Heating the circumference of the $\mathrm{CP}$ induces the optical path change in the $\mathrm{CP}$ as a result of the thermo-optic effect. The $\mathrm{CP}$ thermal lens has a similar profile to a negative lens, which counteracts the positive lens in the test mass due to absorption of the high optical power laser beam, as shown schematically in Fig. 3. Here, the thermal lensing in the $\mathrm{CP}$ is negligible due to the fairly low optical absorption. ${ }^{20} \mathrm{We}$ ignored the contribution of the relatively small thermal expansion effect, which is only $\sim 6 \%$ of the thermo-optic effect. ${ }^{10}$

Due to the low thermal conductivity of fused silica, the thermal time constant for the $\mathrm{CP}$ to reach a steady state tem-

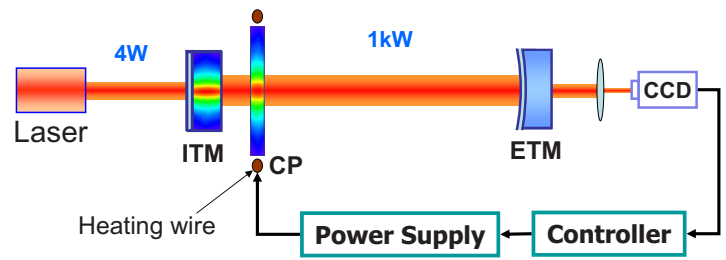

FIG. 2. (Color online) A schematic of the experimental setup at the HOPF at Gingin. A FP cavity consists of two suspended sapphire mirrors, separated by $\sim 80 \mathrm{~m} . \mathrm{A} \sim 4 \mathrm{~W}$ Nd:YAG laser is injected and phase locked into the FP cavity. The CP is inserted inside the cavity close to the ITM. The CCD located behind the ETM is used to measure the transmitted beam profile. The controller produces an error signal from the measured beam diameter change and this is used to control the heating power applied to the heating wire of the $\mathrm{CP}$.

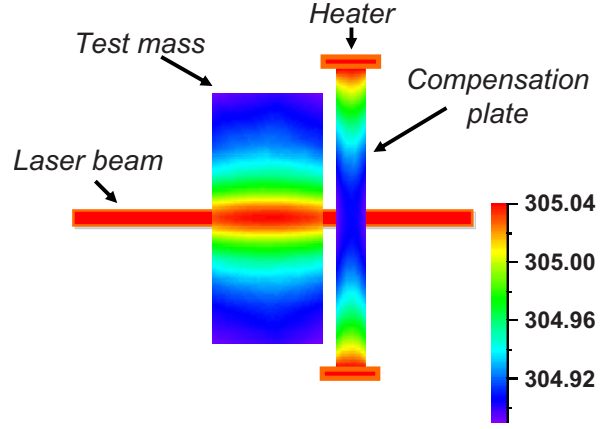

FIG. 3. (Color online) A schematic based on a finite element model for correcting strong thermal lensing in the test mass by circumferential heating of a CP. The color scale indicates temperature in the sapphire test mass, the temperature in the $\mathrm{CP}$ is much higher. The $\mathrm{CP}$ is placed near the sapphire test mass and generates an opposite thermal gradient to the thermal lens in the test mass.

perature gradient is very large, about few thousands of seconds. In order to analyze this heating process, we built a thermodynamic model of the CP by using the ANSYS finite element modeling package and evaluated the time evolution of the thermal focal length of the CP. Figure 4(a) shows the temperature distribution inside the $\mathrm{CP}$ substrate after heating for $2 \mathrm{~h}$ with $5 \mathrm{~W}$ heating power. Figure 4(b) shows the time evolution of the inverse of the focal length of the CP. We see growth of the negative lens. Quasisteady state is achieved in about $1.5 \mathrm{~h}$. The solid curve is the exponential fit with a time constant $\sim 1000 \mathrm{~s}$. The relatively large diameter $(160 \mathrm{~mm})$ of the CP compared to the beam spot size $(\sim 17 \mathrm{~mm})$, combined with the low thermal conductivity of fused silica result in very large phase lag between sensing and actuation. We can see in Fig. 4(b) that the CP is nearly flat in the first 5 min heating. We approximate this to $1 / f=0$ in the curve fitting. The inverse of the focal length $y$ was expressed analytically as

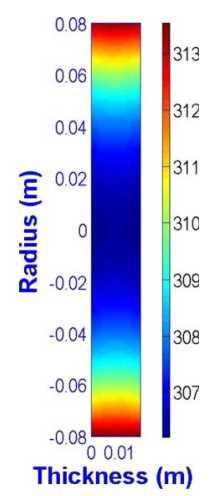

(a)

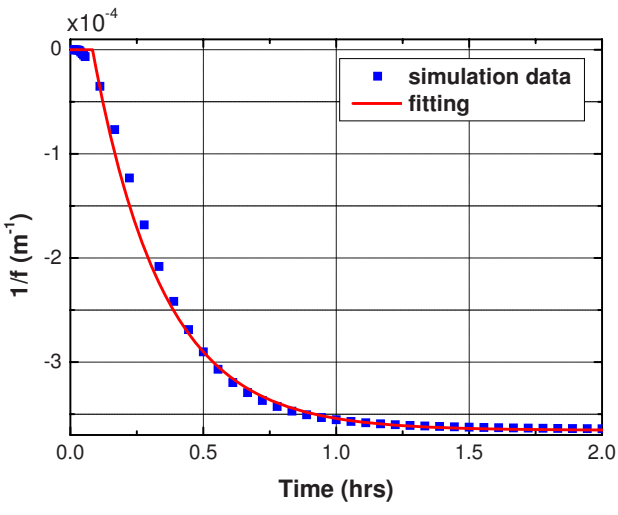

(b)
FIG. 4. (Color online) (a) Temperature distribution inside the CP substrate, the simulation result of applying $5 \mathrm{~W}$ of heating power for $2 \mathrm{~h}$. (b) Time evolution of the inverse of the focal length of the CP. The blue dotted curve is the simulated data. The solid curve is the exponential fitting result. The negative value represents a concave lens. The graph shows that this is a slow process due to the small thermal conductivity of the $\mathrm{CP}$. 


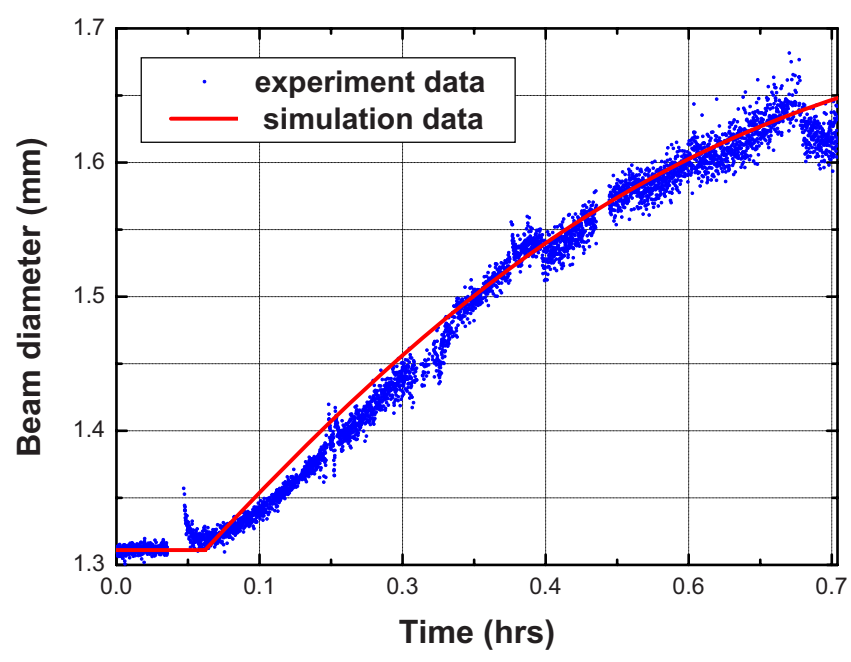

FIG. 5. (Color online) The time evolution of the transmitted beam diameter due to $\sim 10 \mathrm{~W}$ heating power applied to the CP. The blue dots are the experimental data measured by the CCD camera. The dips and peaks in the data occur when the cavity lost lock and was relocked. The red solid line is the simulation result.

$$
y(t)= \begin{cases}0, & t \leq t_{0} \\ A\left(e^{\left[-\left(t-t_{0}\right) / \tau\right]}-1\right), & t>t_{0},\end{cases}
$$

where $A$ is the amplitude, $\tau$ is the time constant, and $t_{0}$ is the delay time. The partner function of $y(t)$ in the $s$-domain after taking the Laplace transform becomes

$$
Y(s)=\frac{-A a}{s(s+a)} e^{-s t_{0}}, \quad a=1 / \tau .
$$

The exponential factor $e^{-s t_{0}}$ is due to the time shifting of $t_{0}$. $Y(s)$ is the key equation for the simulation of the feedback control system. We will discuss it in Sec. III.

\section{EXPERIMENTAL RESULTS AND SIMULATION OF THE CLOSED-LOOP CONTROL}

\section{A. Heating CP experiment}

The thermal lensing effect created by the high optical power induced a change in the beam spot size. This change was measured $^{17}$ by using the CCD camera as shown in Fig. 2. The beam radius $\omega$ on the ETM can be expressed as follows: ${ }^{21}$

$$
\omega=\sqrt{\frac{R_{2} \lambda}{\pi}}\left(\frac{L}{R_{2}-L}\right)^{1 / 4}\left(1+\frac{R_{2}}{R_{1}}\right)^{-1 / 4} \text { for } R_{1} \gg R_{2},
$$

where $\lambda$ is the laser beam wavelength, $L$ is the cavity length, and $R_{1}$ and $R_{2}$ are the radius of curvature of the ITM and ETM, respectively. For the AIGO FP cavity, the ITM is flat in the cold case, i.e., $R_{1}=\infty$. With $\sim 4 \mathrm{~W}$ input laser power, the circulating power reaches $\sim 1 \mathrm{~kW}$. Then the $R_{1}$ became $\sim 2.5 \mathrm{~km}$ due to the laser beam heating the ITM substrate, which in turn decreased the beam spot size on ETM. In the same way, heating the $\mathrm{CP}$ also induces a beam size change in the cavity. Figure 5 shows the time trace of the transmitted beam spot size variation after applying about $10 \mathrm{~W}$ heating power to the CP. The beam spot size at the CCD is about 0.1 of the spot size on the ETM. The blue dotted curve is the experimental result for the transmitted beam diameter

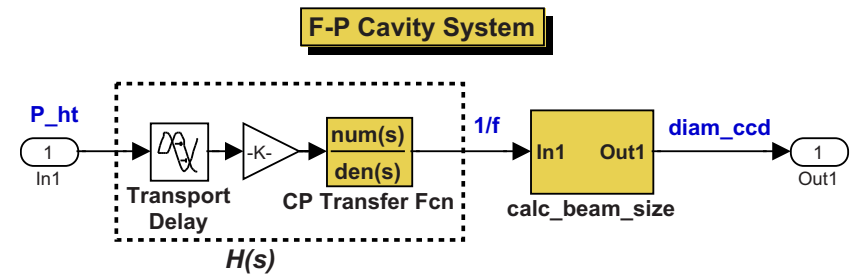

FIG. 6. (Color online) Schematic of a FP cavity system modeled in SIMULINK of MATLAB. The system contains the transfer function model $H(s)$ and a beam size calculation block used to compute the beam spot size corresponding to the change of the focal length change of the $\mathrm{CP}$.

change. Due to the thermal lens of the ITM alone, with $\sim 1 \mathrm{~kW}$ circulation power, the transmitted beam diameter measured at the CCD was changed from 1.56 to $1.31 \mathrm{~mm}$ in a very short time, $\sim 2 \mathrm{~min}$. The heating system began to heat the CP at $t=0$ ( $t$ is the time scale denoted in the figure). The beam spot size had minimal change in the first $5 \mathrm{~min}$, and then began to increase exponentially for about $20 \mathrm{~min}$ before beginning to stabilize due to radiative heat lose. The spikes in the experimental curve were due to losing and regaining cavity lock.

The solid curve in Fig. 5 is the analytical time evolution of the transmitted beam size. It was simulated with a virtual FP cavity system modeled in SIMULINK. ${ }^{22}$ The FP cavity model is shown in Fig. 6. It contains a CP transfer function model and a beam size calculation block. The transfer function model $H(s)$ was obtained based on the Eq. (2) assuming that the input heating power is a step function of time

$$
H(s)=\frac{Y(s)}{1 / s} .
$$

The transport delay block in Fig. 6 denotes the time delay $e^{-s t_{0}}$ in Eq. (2). The $H(s)$ model evaluates the focal length of $\mathrm{CP}$ as a function of the input heating power and the heating time. This change in focal length of the $\mathrm{CP}$ changes the radius of curvature of the combined mirror $(\mathrm{ITM}+\mathrm{CP})$, which in turn changes the beam spot size of the cavity mode. We ignored the change in the intracavity power, which was only $\sim 0.2 \%$ power loss owing to mode's mismatch between the input beam mode and cavity mode, ${ }^{10}$ i.e., the thermal lens of the ITM kept constant. The beam size calculation block uses Eq. (3) to compute the transmitted beam spot diameter corresponding to the value measured by the CCD. $R_{1}$ used here is the radius of curvature of the combined mirror $(\mathrm{ITM}+\mathrm{CP})$. The solid line in Fig. 5 is the time dependent change in the beam size with $5 \mathrm{~W}$ input heating power. The heating power value in the simulation is about half of that used in the experiment. This is a result of using only the ideal thermal absorption in the simulation and ignoring thermal losses. In practice there were insulated multilayers Teflon tapes wrapped around the circumference of the CP between the heating wire and the CP. The heating wire was not in thermal equilibrium with the CP circumference and a significant fraction of heating power was radiated.

\section{B. Feedback control experiment}

We performed the experiment discussed above, and demonstrated closed-loop feedback control of thermal lens- 


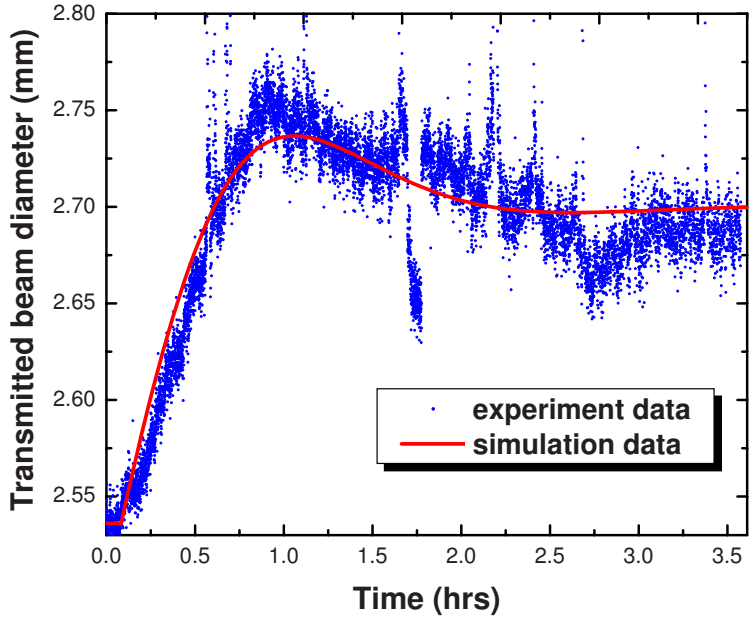

(a)

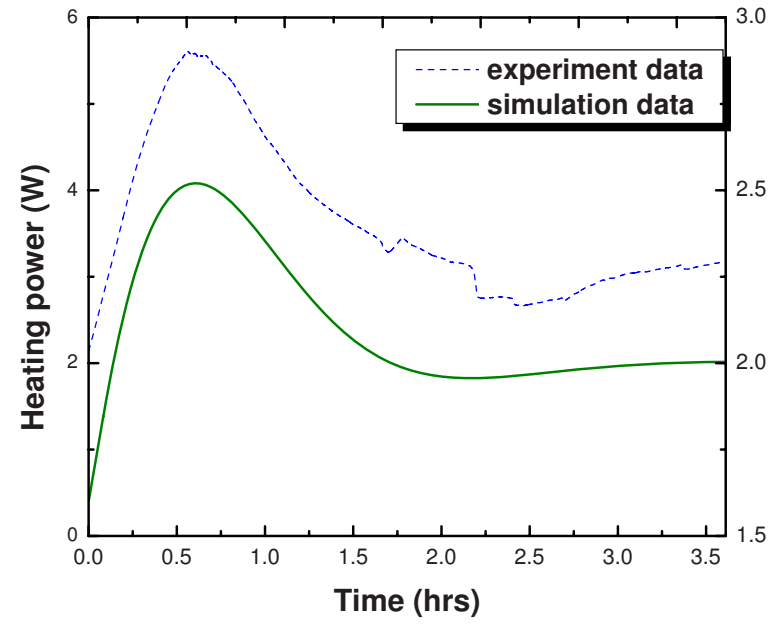

(b)

FIG. 7. (Color online) (a) The time evolution of the transmitted beam size as the feedback control system was applied. The blue dots are experimental data while the solid curve is the analytical result evaluated in SIMULINK. (b) A dash plot of measured time dependence of the heating power applied onto the CP. The solid curve is the simulation result, which shows the heating power is about half of the practical power, agreement with previous analysis.

ing compensation. The results are shown in Fig. 7. Figure 7 (a) shows the time evolution of the transmitted beam diameter. The heating started at $t=0$ (time scale shown in the figure), and achieved stable thermal equilibrium about $3.5 \mathrm{~h}$ later. The transmitted beam diameter finally reached the desired beam spot size $2.7 \mathrm{~mm}$, corresponding to $\sim 17 \mathrm{~mm}$ diameter at the ETM. The beam spot size is different from that in Fig. 5 due to a different distance between the lens and the CCD camera. Figure 7(b) shows the time dependence of the heating power applied to the CP. It shows that the heating power is adequate to compensate the thermal lensing effect in current FP cavity system in HOPF. The experimental heating power curve shows that deviations between $\sim 1.7$ and $\sim 2.5 \mathrm{~h}$ are due to the cavity losing lock and being relocked.

We also created a close-loop control model in SIMULINK to simulate the feedback control loop, as shown in Fig. 8. The system is stable with about $70^{\circ}$ phase margin. Unit gain occurs at $\sim 5.3 \times 10^{-5} \mathrm{~Hz}$. The model consists of the FP cavity system and a controller subsystem. The controller block contains a simple proportional-integral controller used to control the transmitted beam spot size by varying the heating power applied to the CP. Its input signal is the error signal which is the difference between the real beam size and the reference value. The output signal is the heating power applied to the CP. The results from the simulation model are in good general agreement with the experimental data. The simulated beam diameter trace is plotted as solid curve in

Feedback control of thermal lensing compensaton

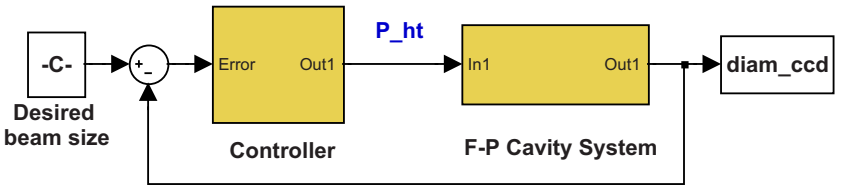

FIG. 8. (Color online) Schematic of the feedback control system. The system includes the FP cavity system and a controller to tune the heating power to maintain the beam size at a desired value.
Fig. 7(a), and the heating power plotted as the solid curve in Fig. 7(b). As previously analyzed, due to the neglect of the thermal losses, the simulated heating power value is approximately two times less than the actual one. The beam diameter reached its peak value after $\sim 1 \mathrm{~h}$ heating, hereafter maintaining a value of $2.7 \mathrm{~mm}$.

\section{CONCLUSION AND FUTURE WORK}

In conclusion, we have studied the heating procedure of the fused silica $\mathrm{CP}$, and demonstrated a negative feedback control system for thermal lensing compensation through monitoring the transmitted beam size change. There are several problems with the method used here, which need not affect its application in full scale interferometer. First, we found that great care was needed to ensure that the $\mathrm{CP}$ was accurately centered on the cavity beam. Otherwise heating the $\mathrm{CP}$ induces a deflection of the beam which in turn affects the circulating power as well as the beam spot size. Second, the direct circumferential heating of the $\mathrm{CP}$ using resistance wire could introduce seismic noise through nonideal antireflective coatings. A heating method such as $\mathrm{CO}_{2}$ laser heating is far more flexible. Still, the importance of careful coalignment of the test mass and the compensating lens is critical in avoiding compensation induced distortions.

An off-axis Hartmann wavefront sensor has been installed and demonstrated on HOPF. ${ }^{23,24}$ In the next stage, we plan to achieve automatic control by using the Hartmann sensor to monitor the thermal lensing and thermal compensation in both the test mass and the CP. Another potential problem relates to the link between thermal lensing and parametric instabilities. ${ }^{25}$ Such instabilities have been recognized as a potential risk for advanced laser interferometric gravitational wave detectors. The parametric gain can be adjusted by tuning the frequency spacing between the optical modes through changing the radius of curvature of one mirror of the cavity. ${ }^{26}$ We have observed three-mode interactions by thermally tuning the radius of curvature of the $\mathrm{CP}^{27} \mathrm{~A}$ closed- 
loop system such as the one we have demonstrated could be used to adjust and maintain the $\mathrm{CP}$ at a certain thermal focal length. However the long thermal time constants mean that dynamical changes in time scales below the time constant cannot be controlled and slow radius of curvature changes are likely to occur for $\sim 1000 \mathrm{~s}$ after the interferometer input power is altered. This could cause the interferometer to pass through regions of parametric instability. On long time scales, thermal compensation offers a possible way to reduce the parametric gain, by allowing the effective radius of curvature to be controlled.

\section{ACKNOWLEDGMENTS}

This project was conducted at the High Optical Power Facility (HOPF) located at Gingin in Western Australia, which was developed in collaboration between the Australia Consortium for Gravitational Astronomy and the U.S. LIGO Laboratory. We would like to thank the International Advisory Committee of the Gingin Facility for their encouragement and advice. This research was supported by the Australian Research Council and the Department of Education, Science and Training and by the U.S. National Science Foundation, through LIGO participation in the HOPF.

${ }^{1}$ D. Shoemaker, LSC meeting, August 2005 (unpublished), Paper No. LIGO-G050386-00-R.

${ }^{2}$ F. Acernese, P. Amico, N. Arnaud, D. Babusci, R. Barille, F. Barone, L. Barsotti, M. Barsuglia, F. Beauville, M. A. Bizouard, C. Boccara, and F. Bondu, Class. Quantum Grav. 21, S385-S394 (2004).

${ }^{3}$ J. Hough, LSC meeting, August 2005 (unpublished), Paper No. LIGOG050419-00-Z

${ }^{4}$ TAMA Collaboration, R. Takahashi, Class. Quantum Grav. 21, S403-S408 (2004).

${ }^{5}$ P. Fritschel, http://www.ligo.caltech.edu/docs/T/T010075-00.pdf.

${ }^{6}$ K. Kuroda, LSC meeting, August 2005 (unpublished), Paper No. LIGOG050412-00-Z.

${ }^{7}$ For Case Statement for Proposed Stage 2 of Gravitational Wave Observatory, see http://www.gravity.uwa.edu.au/docs/AIGOII.pdf.

${ }^{8}$ W. Winkler, K. Danzmann, A. Rüdiger, and R. Schilling, Phys. Rev. A 44,
7022 (1991).

${ }^{9}$ K. A. Strain, K. Danzmann, J. Mizuno, P. G. Nelson, A. Rüdiger, R. Schilling, and W. Walker, Phys. Lett. A 194, 124 (1994).

${ }^{10} \mathrm{R}$. Lawrence, "Active Wavefront Correction in Laser Interferometric Gravitational Wave Detectors," Ph.D. thesis, MIT, 2003.

${ }^{11}$ J. Degallaix, C. Zhao, L. Ju, and D. Blair, Appl. Phys. B: Lasers Opt. 77, 409 (2003).

${ }^{12}$ R. Lawrence, D. Ottaway, M. Zucker, and P. Fritschel, Opt. Lett. 29, 2635 (2004).

${ }^{13}$ R. Lawrence, M. Zucker, P. Fritschel, P. Marfuta, and D. Shoemaker, Adaptive thermal compensation of test masses in Advanced LIGO, LIGOP010023-00-R.

${ }^{14}$ M. Smith, D. Ottaway, and P. Willems, Heating Beam Pattern Optical Design CO2 Laser Thermal Compensation Bench, LIGO-T040057-00.

${ }^{15}$ S. Ballmer, "LIGO interferometer operation at design sensitivity with application to gravitational radiometry," Ph.D. thesis, MIT, 2006.

${ }^{16}$ J. Degallaix, "Compensation of strong thermal lensing in advanced interferometric gravitational waves detectors," Ph.D. thesis, UWA, 2006.

${ }^{17}$ C. Zhao, J. Degallaix, L. Ju, Y. Fan, D. G. Blair, B. J. J.Slagmolen, M. B. Gray, C. M. Mow Lowry, D. E. McClelland, D. J. Hosken, D. Mudge, A. Brooks, J. Munch, P. J. Veitch, M. A. Barton, and G. Billingsley, Phys. Rev. Lett. 96, 231101 (2006).

${ }^{18}$ B. J. J. Slagmolen, High Optical Power Test Facility, ACIGA workshop, April 2004.

${ }^{19}$ R. W. P. Drever, J. L. Hall, F. V. Kowalski, J. Hough, G. M. Ford, A. J. Munley, and H. Ward, Appl. Phys. B: Lasers Opt. 31, 97 (1983).

${ }^{20}$ S. Hild, H. Lück, W. Winkler, K. Strain, H. Grote, J. Smith, M. Malec, M. Hewitson, B. Willke, J. Hough, and K. Danzmann, Appl. Opt. 45, 7269 (2006).

${ }^{21}$ A. E. Siegmann, Lasers (University Science Books, Sausalito, California, 1986).

${ }^{22}$ MATLAB online help, http://www.mathworks.com/.

${ }^{23}$ A. Brooks, P. J. Veitch, J. Munch, and T. L. Kelly, Gen. Relativ. Gravit. 37, 1575 (2005).

${ }^{24}$ A. Brooks, D. Hosken, J. Munch, P. J.Veith, Z. Yan, C. Zhao, Y. Fan, J. Li, D. Blair, and P. Willems, http://www.ligo.caltech.edu/docs/ScienceDocs/ P/P080089-00.pdf.

${ }^{25}$ V. B. Braginsky, S. E. Strigin, and S. P. Vyatchanin, Phys. Lett. A 287, 331 (2001)

${ }^{26}$ C. Zhao, L. Ju, J. Degallaix, S. Gras, and D. G. Blair, Phys. Rev. Lett. 94, 121102 (2005).

${ }^{27}$ C. Zhao, L. Ju, Y. Fan, S. Gras, B. J. J. Slagmolen, H. Miao, P. Barriga, D. G. Blair, D. J. Hosken, A. F. Brooks, P. J. Veitch, D. Mudge, and J. Munch, Phys. Rev. A 78, 023807 (2008). 
Review of Scientific Instruments is copyrighted by the American Institute of Physics (AIP). Redistribution of journal material is subject to the AIP online journal license and/or AIP copyright. For more information, see http://ojps.aip.org/rsio/rsicr.jsp 